\title{
Detection and Development on Total Flavonoids by the Way of $\mathrm{Al}\left(\mathrm{NO}_{3}\right)_{3}$ Ultraviolet Colorimetry
}

\author{
Du Qing ${ }^{1 *}$, Li Wenyuan², Yang Shibin² ${ }^{2}$ Duo Jie ${ }^{3}$, Li Qien ${ }^{3 *}$ and Xue Yizhuo ${ }^{1}$
}

${ }^{1}$ Key Laboratory for Tibet Plateau Phytochemistry of Qinghai Province, College of Pharmacy, Qinghai Nationalities University, Xining, 810007, China

${ }^{2}$ Department of Pharmacy, Qinghai Medical College, Qinghai University, Xining 810016, China

ISSN: 2577-1914

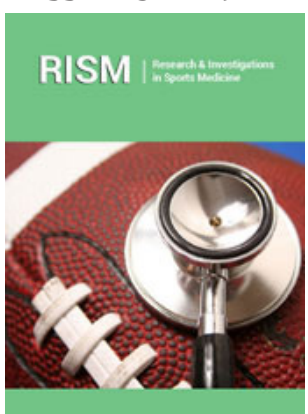

*Corresponding author: Du Qing, No.3, Bayi mid-road,East of city Dist, Xining City, Qinghai Province, 810007, China Li Qien, No.16, Kunlun Road, North of city Dist, Xining City, Qinghai Province, 810007, China

Submission: 梅 September 29, 2020

Published: 眥October 15, 2020

Volume 7 - Issue 2

How to cite this article: Du Qing, Li Wenyuan, Yang Shibin, Duo Jie, Li Qien, Xue Yizhuo. Detection and Development on Total Flavonoids by the Way of $\mathrm{Al}\left(\mathrm{NO}_{3}\right)_{3}$ Ultraviolet Colorimetry. Res Inves Sports Med, 7(2), RISM.000658. 2020. DOI: 10.31031/RISM.2020.07.000658

Copyright@ Du Qing, Li Qien, This article is distributed under the terms of the Creative Commons Attribution 4.0 International License, which permits unrestricted use and redistribution provided that the original author and source are credited.
${ }^{3}$ State Key Laboratory of Tibetan medicine research and development, Tibetan Medical College of Qinghai University, Xining, 810016, China

\begin{abstract}
Flavonoids are mainly distributed in higher plants and almost exist in all green plants of Traditional Chinese Medicine (TCM). In this paper we detect the total flavonoids from the standards and samples arising from special flavonoids frameworks by the way of $\mathrm{Al}\left(\mathrm{NO}_{3}\right)_{3}$ ultraviolet colorimetry so as to analyze the features of wavelength degree and peak strength in the graph of ultraviolet spectrum. The diverse positions and strengths of absorption band $200-600 \mathrm{~nm}$ in the ultraviolet spectrum are distinct taking the rutin reference and total flavonoids from Corydalis conspersafor instance. We can find there are 4 pairs peaks \& peak valleies (325 and 335; 345 and 355; 360 and 365; 375 and 380) between $300 \mathrm{~nm}$ and $400 \mathrm{~nm}$ through scanning the rutin standard solution. There is one strong peak at the $242 \mathrm{~nm}$ and one weak peak at about $510 \mathrm{~nm}$. The important result is that the absorbance always is highest at the $345 \mathrm{~nm}$ and the absorbance is from 0.133 to 1.487. After that, we put forward some thoughts for the further researches for the verification of chemical and biochemical reaction mechanism by the Al(NO3)3 ultraviolet colorimetry, which can be used for the assay of flavonoids with special structures from the diverse families.
\end{abstract}

Keywords: Flavonoids; Structure and origin; Characteristics of UV; Rutin; $\mathrm{Al}\left(\mathrm{NO}_{3}\right)_{3}$ Ultraviolet colorimetry

\section{Introduction}

The discovery of flavonoids has the long-standing and abundant history. In the 1930s, the Hungarian scientists Rusznyak and Szent Györgyi identified a substances which is named 'Citrin' from the fruit of lemon' peels [1]. However, at the beginning the substance was designated as vitamin P (P for permeability), one kinds of the member from the vitamin families. Later Bruckner and Szent Györgyi found it was a mixture of the flavonoid's hesperidin and eriodictyol glucoside including rutin. So far flavonoids compound has been identified for much more than 6000 varieties from all kinds of plants [2].

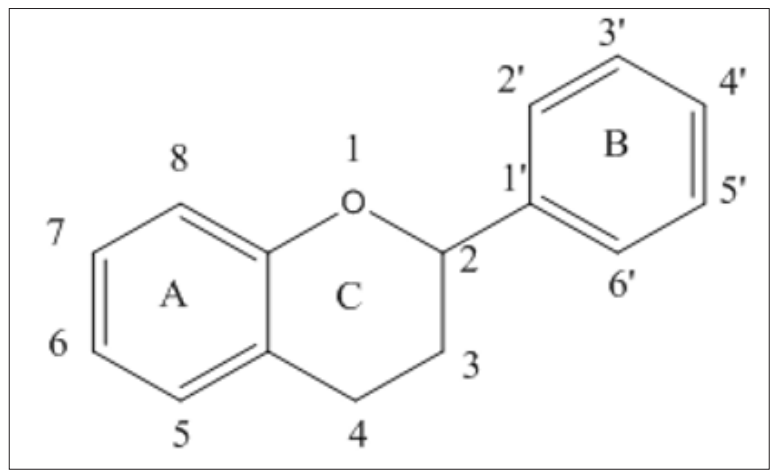

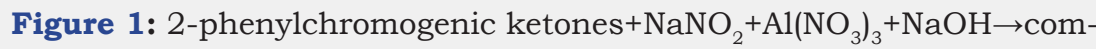
plex structure?

Flavonoids are a class of compounds widely existing and distributed in nature, with 2-phenylchromone (flavone) and $\mathrm{C}_{6}-\mathrm{C}_{3}-\mathrm{C}_{6}$ framework compounds (Figure 1). There is a keto carbonyl group in their molecules. The oxygen atom on the first position is alkaline and can 
form salt with strong acid. Flavonoids have diverse pharmacological activities such as antibacterial [3], antiallergic [4], anticancer [5] and antiviral [6] activities etc. reported in the TCM.

In general, the UV absorption spectrum of most flavonoids in methanol consists of two main absorption bands. The absorption band I (Cinnamoyl ring) is between 300 500 nm and the absorption band II (Benzoyl ring) is between 200 280nm [7]. If aluminum salt was added, $\mathrm{Al}^{3+}$ formed a stable complex with flavonoids, and band I moved significantly to the long wavelength direction (red shift); in the strong alkaline solution of $\mathrm{NaNO}_{2}$, its absorbance value was the highest at $510 \mathrm{~nm}$ [8]. Therefore we can infer the structures of various flavonoids for these changes in detailed and detect the contents of the flavonoids if we can carry out the researches laws regarding the special structures flavonoids by $\mathrm{Al}\left(\mathrm{NO}_{3}\right)_{3}$ Ultraviolet colorimetry.

\section{Materials and Methods}

\section{Collection and treatment of Corydalis conspersa}

The plants were collected in Jigang mountain, Huangnan Prefecture, Qinghai Province, in August 2017 and identified by Professor Lin Pengcheng (Qinghai Nationalities University) identified as a Tibetan medicine Corydalis conspersa [9]. After cleaning, the samples were divided into roots, stems, leaves and flowers, then dried in the shade, crushed through 80 mesh sieves for further study.

\section{Materials}

Rutin standard substance (HPLC, purity $\geq 98 \%$, Shanghai Yuanye Biotechnology Co., Ltd); Ethanol (analytical pure, Tianjin Fuyu Fine Chemical Co., Ltd); Purified water (Hangzhou Wahaha Group Co., Ltd.); Nitrite Sodium (AR, Yantai Shuangshuang Chemical Co., Ltd); Aluminum nitrate (Shanghai Guangnuo Chemical Technology Co., Ltd.); Sodium hydroxide(Chinese Medicine Group Chemical Reagent Co., Ltd.).

\section{Instruments}

UV -Vis2450 spectrophotometer (Japan island); Cuvette (Shimadzu, Japan); HH-6 digital display constant temperature water bath pan (Changzhou Aohua Instrument Co., Ltd.); AL204 electronic balance (METTLER TOLEDO Instrument Co., Ltd., Switzerland); DFT-100 high speed powder (Shanghai Dingguang Machinery Equipment Co., Ltd.)

\section{Preparation of standard substance solutions}

Weigh $10.9 \mathrm{mg}$ rutin standard, add appropriate amount of $60 \%$ ethanol, heat to dissolve, and fix the volume in a $100 \mathrm{ml}$ volumetric flask after room temperature to prepare rutin standard with the concentration of $0.1 \mathrm{mg} \bullet \mathrm{ml}^{-1}$. Shake well and set aside. Take an appropriate amount of rutin standard solution and scan it in the range of 200-700nm to observe the absorption wavelength of rutin [9].

\section{Preparation of sample solutions}

The root powder of $C$. conspersa was taken, and $0.1 \mathrm{~g}$ powder was precisely weighed ( 3 times were determined in parallel under each experimental condition), and then put into a $250 \mathrm{ml}$ flask with a condensation tube, put it in a constant temperature water bath, add a $30 \mathrm{ml}, 40 \mathrm{ml}, 50 \mathrm{ml}, 60 \mathrm{ml}$ amount of $60 \%$ ethanol to extract total flavone, and keep the volume to $100 \mathrm{ml}$ volumetric flask using the $60 \%$ ethanol for standby.

\section{Detection of absorption wavelength conditions}

Take $2.0 \mathrm{ml}, 4.0 \mathrm{ml}, 6.0 \mathrm{ml}, 8.0 \mathrm{ml}, 10.0 \mathrm{ml}$ rutin standard and $0.5 \mathrm{ml}$ sample solution respectively, add $2.5 \mathrm{ml}$ of $5 \%$ sodium nitrite solution, $2.5 \mathrm{ml}$ of $10 \%$ aluminum nitrate solution, $20.0 \mathrm{ml}$ of $4 \%$ sodium hydroxide solution, and finally add $60 \%$ ethanol solution to fix the volume to $50.0 \mathrm{ml}$. After adding reagent in each step above, ultrasonic wave for $5 \mathrm{~min}$, shake well, and stand for $15 \mathrm{~min}$. The absorbance was measured at different wavelength with a $1.0 \mathrm{~cm}$ quartz cuvette, recording the spectra and peaks [9]. The reaction equation is as follows (1).

\section{Results}

\section{Rutin standard substance solutions}

From Figure 2, we can find there are 8 peaks and peak valleys between $300 \mathrm{~nm}$ and $400 \mathrm{~nm}$, at the same time there is one strong peak at the $242 \mathrm{~nm}$ and one weak peak at about $510 \mathrm{~nm}$. We summary the wavelength position and absorption of the peaks in Table 1. It shows that the absorbance is changing up and down because the 4 pairs peaks \& peak valleies (325 and 335; 345 and $355 ; 360$ and $365 ; 375$ and 380 ) with the different wavelengths at the same concentration. The important result is that the absorbance always is highest at the $345 \mathrm{~nm}$ of the same concentration and the absorbance is between 0.133 and 1.487 .

Table 1: The average absorbance of standard solution from the corresponding concentration and diverse wavelength (three parallel repetitions).

\begin{tabular}{|c|c|c|c|c|c|c|c|c|c|}
\hline \multirow{2}{*}{ Concentration(mg/ml) } & \multicolumn{9}{|c|}{ Different wavelengths and absorbance } \\
\cline { 2 - 10 } & $\mathbf{3 2 5}$ & $\mathbf{3 3 5}$ & $\mathbf{3 4 5}$ & $\mathbf{3 5 5}$ & $\mathbf{3 6 0}$ & $\mathbf{3 6 5}$ & $\mathbf{3 7 5}$ & $\mathbf{3 8 0}$ \\
\hline 0.00436 & 0.231 & 0.277 & 0.287 & 0.271 & 0.247 & 0.239 & 0.191 & 0.133 \\
\hline 0.00872 & 0.449 & 0.484 & 0.658 & 0.648 & 0.586 & 0.52 & 0.428 & 0.302 \\
\hline 0.01308 & 0.569 & 0.623 & 0.85 & 0.873 & 0.786 & 0.703 & 0.566 & 0.404 \\
\hline 0.01744 & 0.742 & 0.79 & 1.223 & 1.136 & 1.037 & 0.844 & 0.677 & 0.5 \\
\hline 0.0218 & 0.907 & 0.904 & 1.487 & 1.376 & 1.199 & 1.018 & 0.81 & 0.567 \\
\hline
\end{tabular}




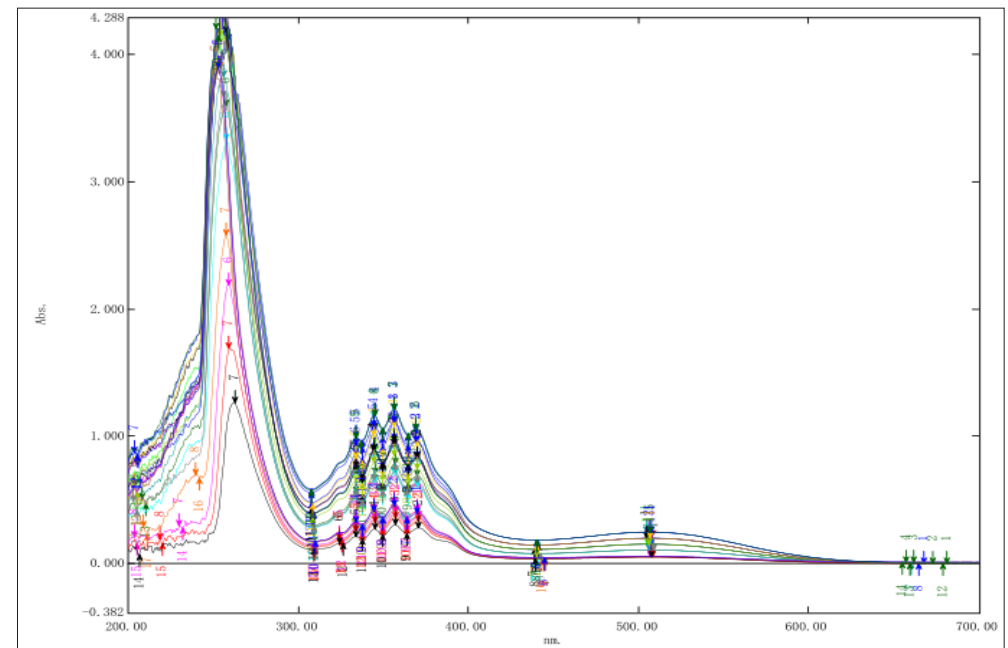

Figure 2: The UV scanning graph of rutin standard substance solutions.

\section{Sample solutions}

From Table 2 and Figure 3 compared to the Figure 2, we found that there are the series of peaks \& peak valleies between 310 and
$400 \mathrm{~nm}$ the same as the standard solutions. There shows the highest absorbance while using the material liquid ratio of $50 \mathrm{ml}: 0.1 \mathrm{~g}$ and the most contents of flavonoids.

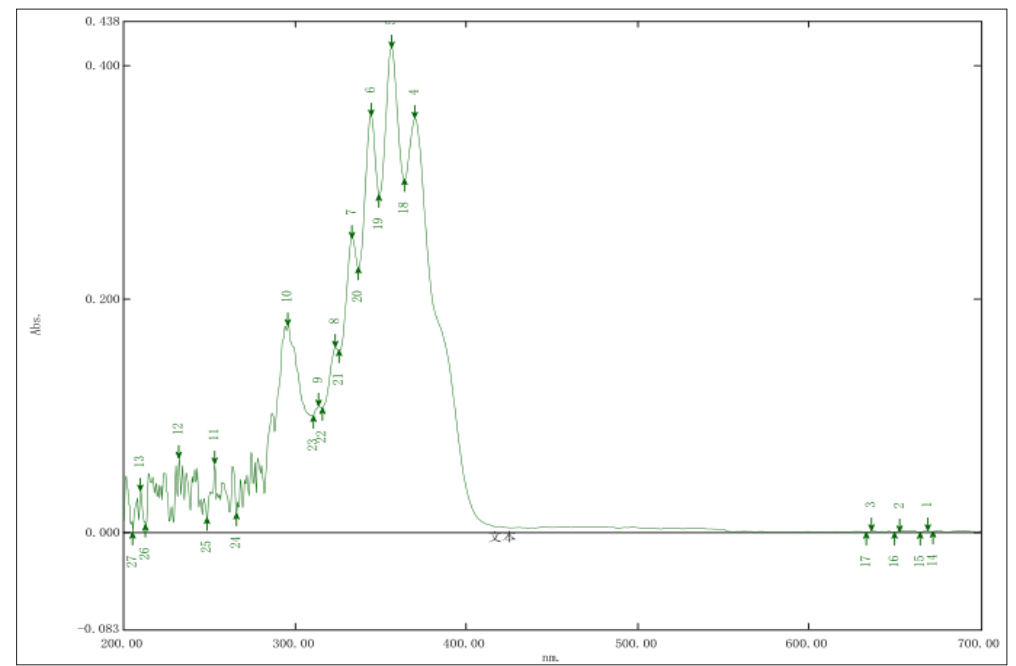

Figure 3: The UV scanning graph of sample solution.

Table 2: The average absorbance of sample solution at $345 \mathrm{~nm}$ wavelength (three parallel repetitions) from the diverse material liquid ratio (30ml:0.1g; 40ml:0.1g; 50ml:0.1g; 60ml:0.1g).

\begin{tabular}{|c|c|c|c|c|}
\hline Times & $\mathbf{3 0}$ & $\mathbf{4 0}$ & $\mathbf{5 0}$ & $\mathbf{6 0}$ \\
\hline 1 & 0.262 & 0.292 & 0.469 & 0.398 \\
\hline 2 & 0.264 & 0.278 & 0.419 & 0.372 \\
\hline 3 & 0.246 & 0.283 & 0.421 & 0.347 \\
\hline Average & 0.257 & 0.284 & 0.43678 & 0.372 \\
\hline
\end{tabular}

\section{Discussion}

The commonly methods used to determine the contents of flavonoids is ultraviolet spectrophotometry, which is mainly used for the determination of total flavonoids, which can be divided into direct determination (determination at the maximum absorption wavelength of UV spectrum compared to the standard reference substance) and metal complex salt colorimetry (refers to the determination of total flavonoids). Flavonoids with specific structure react with aluminum salt, magnesium salt, lead salt, zirconium salt and other reagents to form colored complex, which can be used for the identification and content determination of some types of flavonoids. In this paper, we determinate the total flavonoids through colorimetry of aluminum nitrate or aluminum chloride in alkaline conditions at the maximum absorption wavelength of the colored complex.

The total flavonoids were extracted by ethanol under different conditions, the flavonoids with 5-hydroxy, 3-hydroxy or o-dihydroxy were complexed with $\mathrm{Al}\left(\mathrm{NO}_{3}\right)_{3}$ and $\mathrm{NaNO}_{2}$. After the preliminary 
study, it was found that there were 4 pairs absorption peaks in the range of 300-400nm after adding the complexing reagent. However, some flavonoids have no maximum absorption or weak absorption near $500 \mathrm{~nm}$. Some non-flavonoid substances have maximum absorption or strong absorption near 500nm [10]. Therefore, with rutin as the reference substance and $\mathrm{Al}\left(\mathrm{NO}_{3}\right)_{3}-\mathrm{NaNO}_{2}-\mathrm{NaOH}$ as the chromogenic reagent, the method for the determination of total flavonoids at $510 \mathrm{~nm}$ is not specific and firstly we should scan the treated sample solutions through the UV spectrophotometer to define the detected wavelength having the maximum absorbance.

\section{Conclusion}

It is important to clarify the relationship between the absorption peaks and flavonoids with special structures. We can adopt the below methods to verify to clearly know the reactions courses. Firstly add one kind of reagent as the reaction equation (1) into the standard solution of different structures changing the concentrations of the reagent and scan the solutions using the UV spectrophotometer to summary the laws of graph alterations step by step. It is huge works and how do they complex with each other among groups? Secondly observe and analyze the intensity of absorption peaks because of the altered concentration. Thirdly detect the reaction speeds or changes of graphs through adding or removing the reagents one by one so as to verify the key steps during the reactions process. Finally, we develop the certain combined instruments to auto-operate the reagents to the solution not only improving the accuracy and efficient, but also saving the cost of manual labor and material resources.

The solutions and approaches to all aspects of the problems are the key technical difficulties and challenges in the future researches. We must deeply discover and verify the reaction courses accompany the advanced technology to well explain the relative structures and biochemical reaction mechanisms.

\section{Funding Statement}

This work was supported by Qinghai Tibet Plateau phytochemistry Key Laboratory (2020-ZJ-Y20); Qinghai Provincial Science and Technology Department Project (2016-ZJ-903); Spatial Grid Fusion and Sharing of Traditional Chinese Medicine Resources and Related Basic Data (Fundamental Special Science and Technology Projects(2013FY114500); National Natural Science Foundation of China (31860585); Natural Science Foundation of Qinghai Science \& Technology Department (2019-ZJ-907); the State key laboratory construction project (2015DQ870717).

\section{Acknowledgment}

Give the thanks to the teachers and students as follows: Liu Mingdi, Guo Sucheng, Hou Yongqiang, Zhao Wei, Jiang Cai, Chen Jinfang, Shen Yuexia, Bao Yuting, Huang Shibo, Ren Zhuo, Shu Min, Chen Xi, Li Xinyue, Wu Zesi, Song Yanhong and Li Yifan, Wu Jiang for collecting and processing samples.

\section{References}

1. Ali DM, Wong KC, Lim PK (2005) Flavonoids from Blumea balsamifera. Fitoterapia 76(1): 128-130.

2. Arteaga E, Villaseca P, Rojas A, Marshall G, Bianchi M (2004) Phytoestrogens possess a weak antioxidant activity on low density lipoprotein in contrast to the flavonoid quercetin in vitro in postmenopausal women. Climacteric 7(4): 397-403.

3. Cushnie TPT, Lamb AJ (2011) Recent advances in understanding the antibacterial properties of flavonoids. Int J Antimicrob Agents 38(2): 99-107.

4. Cheong H, Ryu SY, Oak MH, Cheon SH, Yoo GS, et al. (1998) Studies of structure activity relationship of flavonoids for the antiallergic actions. Archive of Pharmacology Research 21(4): 478-480.

5. Divyashree Ravishankar, Amit Kumar Rajora, Francesca Greco, Helen MI Osborn (2013) Flavonoids as prospective compounds for anti-cancer therapy. Int J Biochem Cell Biol 45(12): 2821-2831.

6. Maja Bekut, Snežana Brkić, Nebojša Kladar, Gordana Dragović, Neda Gavarić, et al. (2017) Potential of selected Lamiaceae plants in anti(retro) viral therapy. Pharmacol Res 133: 301-314.

7. Yao XS (2001) Natural Medical Chemistry. Natural medicine press, Beijing, China.

8. Haben JB (1983) Flavonoids. Science Press, Beijing, China.

9. Du Qing, Wu Jiang, Liu Mingdi (2019) Optimization of extraction process of total flavonoids from corydalis conspersa maxim by response surface methodology. Traditional Chinese Drug Research \& Clinical Pharmacology 30(5): 608-613.

10. LV Lin, Tao NP (2009) Determination of total flavonoids content in citrus peels by ultraviolet spectrophotometry. Modern Food Science and Technology 25(2): 217-220. 